\title{
Endometriosis de la pared abdominal: estudio descriptivo de una serie de 21 casos
}

\author{
Abdominal wall endometriosis: A descriptive study of a series of 21 cases \\ Juliana Restrepo ${ }^{1}$, Alvaro Turizo ${ }^{2}$, Sebastián Tabares ${ }^{1}$, Andrés Felipe Ortiz $^{3}$, Alejandro Vélez ${ }^{4}$, \\ Diana Paola Cuesta ${ }^{5}$

\begin{abstract}
Médico, cirujano general, Facultad de Medicina, Universidad Pontificia Bolivariana, Medellín, Colombia Médico, residente de Cirugía General, Facultad de Medicina, Universidad Pontificia Bolivariana, Medellín, Colombia Médico, especialista en Radiología e Imágenes Diagnósticas. Facultad de Medicina, Universidad ICESI. Cali, Colombia Medellín, Colombia
\end{abstract} \\ 4 Médico patólogo. Hospital Pablo Tobón Uribe, Facultad de Medicina, Universidad Pontificia Bolivariana; Laboratorio Dinámica, \\ 5 Médica, Ph.D. en Epidemiología, Facultad de Medicina, Pontificia Universidad Bolivariana, Medellín, Colombia
}

\section{Resumen}

Introducción. La endometriosis de la pared abdominal se define como la presencia de tejido endometrial en cualquiera de las capas que componen la pared abdominal. Su incidencia es baja y se caracteriza por un diagnóstico tardío.

Materiales y métodos. Se trata de un estudio descriptivo y ambiespectivo entre 2010 y 2014 en pacientes con endometriosis de la pared abdominal, cuyo análisis patológico fue realizado en un centro de ayudas diagnósticas de Medellín. Se identificaron las variables histopatológicas del reporte, y la información clínica mediante una entrevista telefónica suministrada por la paciente. Se analizaron los datos con medidas descriptivas de resumen.

Resultados. Participaron 2I de 65 pacientes con diagnóstico de endometriosis de la pared abdominal. La media de edad al momento del diagnóstico fue de 35,3 años (desviación estándar, DE=8), el 71,4 \% tenía el antecedente de cesárea y, el 38,I \%, el de endometriosis pélvica. El 95,2 \% de las pacientes manifestaron dolor, de las cuales el $50 \%$ lo percibió como constante con agudización cíclica y, el 40 \%, como cíclico; además, el 90,5\% manifestó sensación de masa. La mediana del tiempo desde la aparición de la lesión hasta el diagnóstico, fue de 24 meses (RIQ=6-60). Solo en cuatro pacientes se hizo el diagnóstico prequirúrgico. El tratamiento fue quirúrgico en todas las pacientes y ocho $(38, \mathrm{I} \%)$ presentaron recidiva.

Conclusiones. La endometriosis de la pared abdominal usualmente se manifiesta como masas dolorosas asociadas con cicatrices quirúrgicas previas, generalmente de origen ginecológico y los síntomas empeoran con la menstruación. Es usual que su diagnóstico sea tardío y pocas veces se hace antes del estudio histopatológico. El tratamiento de elección es la resección quirúrgica, aunque no es despreciable el porcentaje de recidivas.

Palabras clave: endometriosis; pared abdominal; ombligo; epidemiología; procedimientos quirúrgicos.

Fecha de recibido: 23/05/2019 - Fecha aceptación: 29/07/2019

Correspondencia: Juliana Restrepo, Calle 46 N5-21, Bogotá, D.C., Colombia

Teléfono: (57) (316) 345-0845, teléfono: julirpo85@gmail.com

Citar como: Restrepo J, Turizo A, Tabares S, Ortiz AF, Vélez A, Cuesta DP. Endometriosis de la pared abdominal: estudio descriptivo de una serie de 21 casos. Rev Colomb Cir. 219;34:338-45. https://doi.org/10.30944/20117582.514.

Este es un artículo de acceso abierto bajo una Licencia Creative Commons - BY-NC-ND https://creativecommons.org/licenses/by-nc-nd/4.0/deed.es 


\begin{abstract}
Introduction: Abdominal wall endometriosis is defined by the presence of endometrial tissue in any of the layers that compose the abdominal wall. It has a low incidence and is characterized by a late diagnosis.

Materials and Methods: A descriptive, ambispective study that included patients with abdominal wall endometriosis whose pathological analysis was performed in a diagnostic center in Medellín between 2010 and 2014. Histopathological variables of the report were identified, and clinical information was provided by the patient by a phone interview. They were analyzed with descriptive summary measures.

Results: 2I patients with abdominal wall endometriosis of 65 identified participated. The mean age at diagnosis was 35.3 years $\pm 8,7 \mathrm{I} .4 \%$ had a prior caesarean section and 38.I\% had pelvic endometriosis. $95.2 \%$ manifested pain, among them, $50 \%$ was perceived as constant with cyclical exacerbation, $40 \%$ cyclical; $90.5 \%$ manifested mass sensation. The median from the onset of the lesion to the diagnosis was 24 months (IQR 6-60). Only four patients had pre-surgical. The treatment was surgical in all patients and eight (38.I\%) had recurrence.

Conclusions: Abdominal wall endometriosis usually manifests through painful masses associated with previous surgical scars usually of gynecological origin and whose symptoms worsen with menstruation. Its diagnosis is usually late and it is rarely reached before the histopathological study. Management of choice is surgical resection, however, its percentage of recurrence is not negligible.
\end{abstract}

Key words: endometriosis; abdominal wall; umbilicus; epidemiology; surgical procedures.

\section{Introducción}

La endometriosis se define como la presencia de tejido endometrial, funcional, situado por fuera de la cavidad uterina ${ }^{1,2}$. Este tejido ectópico usualmente se sitúa en la pelvis, aunque también puede encontrarse en los pulmones, el intestino, los uréteres, el cerebro y la pared abdominal, entre otros sitios ${ }^{\text {. }}$

La endometriosis de la pared abdominal fue descrita por primera vez en $1956{ }^{3}$ y se define como la presencia de tejido endometrial superficial al peritoneo ', que puede afectar desde la dermis, pasando por el tejido celular subcutáneo, hasta la fascia y los músculos de la pared abdominal ${ }^{4}$. Muchos casos de endometriosis de la pared abdominal se han asociado con cirugías ginecobstétricas, como la cesárea; su diagnóstico es difícil y tardío por confundirse con entidades como hernias, hematomas o lipomas ${ }^{\mathrm{I}}$.

Presenta una incidencia reportada muy baja, entre $0,03 \%$ y $3,5 \%$, y corresponde al I, $34 \%$ de las cirugías practicadas por endometriosis 5 . En cuanto a su etiopatogenia, se postula como principal teoría la implantación directa de células endometriales sobre la herida quirúrgica tras una histerotomía; sin embargo, se encuentra descrita también la diseminación por vía hematógena o linfática, y es de tener en cuenta la presencia de casos sin antecedentes quirúrgicos ${ }^{3}$. Usualmente, esta enfermedad no se diagnostica ni se sospecha antes del resultado histopatológico; además, los cirujanos generales son los que más dificultades tienen para llegar a su diagnóstico ${ }^{6}$.

El diagnóstico inicial es clínico, el cual se realiza por la presencia de una masa en la pared abdominal, la mayoría de las veces dolorosa, y se encuentra en asociación con el ciclo menstrual. Cuando exista duda o sea necesario conocer la extensión de la lesión, la ecografía, la tomografía computarizada y la resonancia magnética pueden ser de ayuda, al igual que la biopsia por aspiración con aguja fina (BACAF) si se quiere descartar una neoplasia maligna ${ }^{3}$.

Los implantes de endometriosis pueden sufrir transformación maligna en 0,3 a I \% de los $\operatorname{casos}^{2,6,7}$, mientras que la transformación maligna de lesiones localizadas en la pared abdominal es aún más rara. El más común es el carcinoma de células claras, seguido del carcinoma endometroide ${ }^{\text {? }}$. 
El objetivo de este estudio fue describir las características demográficas, morfológicas y clínicas de una cohorte de pacientes con diagnóstico de endometriosis de la pared abdominal, confirmado por histopatología.

\section{Metodología}

Se llevó a cabo un estudio observacional y ambiespectivo, en una cohorte descriptiva de pacientes con diagnóstico histopatológico de endometriosis de la pared abdominal, hecho en el Laboratorio y Ayudas Diagnósticas, Dinámica, entre 2010 y 2014 en Medellín, Colombia.

Se incluyeron las pacientes con diagnóstico histopatológico de endometriosis en especímenes de piel y tejidos blandos de la pared abdominal, y se excluyeron aquellas que rechazaron participar en el estudio al momento de solicitar el consentimiento informado verbal telefónico o que no fue posible localizar.

De un listado de reportes histopatológicos suministrado por Dinámica en el periodo de estudio, se identificaron las pacientes y su información de contacto. Dos investigadores siguieron un protocolo de llamada y, después de la identificación personal y de informar el propósito del estudio, se solicitó un consentimiento verbal de participación para hacer una entrevista telefónica y registrar la información demográfica, clínica y morfológica de cada paciente.

Se identificaron las siguientes variables de las pacientes: edad, raza, antecedentes personales y familiares; las características de la lesión, como localización, dolor, sensación de masa y secreción de líquido; el tratamiento recibido y la evolución de la enfermedad. Las características de presentación de la lesión se evaluaron según si eran cíclicas (empeoraban durante la menstruación), intermitentes (no relacionadas con el ciclo menstrual), constantes (dolor continuo que no mejoraba ni empeoraba), constantes con agudización cíclica (dolor continuo que empeoraba con la menstruación), o constantes con agudización intermitente (dolor constante que empeoraba en ocasiones, pero no se asociaba con la menstruación). La información fue registrada en un formato de recolección y diligenciada en una base electrónica de datos.

Las variables cualitativas se presentaron con su frecuencia absoluta y proporción, y las variables cuantitativas, con promedio y desviación estándar o mediana y rango intercuartílico según si presentaron distribución normal. Se utilizó el programa estadístico IBM SPP ${ }^{\mathrm{TM}}$, versión I9, para analizar los datos del estudio.

El estudio fue avalado por el Comité de Ética en Investigación en Salud de la Universidad Pontificia Bolivariana.

\section{Resultados}

Se identificaron 65 reportes histopatológicos de pacientes con endometriosis en la pared abdominal en el periodo de estudio. No fue posible localizar a 43 pacientes y una rechazó participar luego de la solicitud del consentimiento verbal. Se analizaron los datos de 2I pacientes.

La edad promedio al momento del diagnóstico fue de 35,3 $\pm 7,5$ años. Doce pacientes (57,I \%) se consideraron de raza mestiza y, nueve $(42,9 \%)$, de raza blanca.

Ocho $(38, \mathrm{I} \%)$ pacientes tenían antecedentes de endometriosis pélvica y, cinco (23,8 \%), antecedentes familiares en primer grado de endometriosis pélvica. Entre los antecedentes patológicos evaluados, se encontraron ocho (38,I \%) pacientes con síndrome de intestino irritable, ocho $(38, \mathrm{I} \%)$ con dispareunia, ocho $(38, \mathrm{I} \%)$ con migraña, cuatro (19\%) con algún tipo de alergia, tres (I4,3\%) con hipotiroidismo, tres (I4,3\%) con asma, tres (I4,3\%) con algún tipo de cáncer o tumor y una paciente con fibromialgia. El 33,3\% presentaba algún otro antecedente, como hipertensión arterial sistémica, dermatitis, síndrome de ovario poliquístico, gastritis, hiperprolactinemia y trasplante renal (tabla I).

En I7 (8I \%) pacientes se reportó, al menos, un embarazo previo al momento del diagnóstico y una mediana del número de embarazos de uno (rango intercuartílico, RIQ=I-3). Como antecedentes quirúrgicos, se registraron: cesárea en I5 $(7 \mathrm{I}, 4 \%)$ pacientes, laparoscopia ginecológica en diez (47,6 \%), histerectomía abdominal en siete $(33,3 \%)$, herniorrafia umbilical en tres ( $14,3 \%)$ y 
Tabla 1. Antecedentes personales en 21 pacientes con endometriosis de la pared abdominal $(\mathrm{N}=21)$

\begin{tabular}{ll}
\hline Antecedentes personales & $\mathbf{n}(\%)$ \\
\hline Dispareunia & $8(38,1)$ \\
Endometriosis pélvica & $8(38,1)$ \\
Síndrome de intestino irritable & $8(38,1)$ \\
Migraña & $8(38,1)$ \\
Antecedentes familiares en primer grado & $5(23,8)$ \\
de endometriosis & $4(19,0)$ \\
Alergias & $3(14,3)$ \\
Asma & $3(14,3)$ \\
Cáncer o tumor & $3(14,3)$ \\
Hipotiroidismo & $2(9,5)$ \\
Disfunción mandibular & $1(4,8)$ \\
Fibromialgia & $0(0,0)$ \\
Cistitis & $7(33,3)$ \\
Otros &
\end{tabular}

episiotomía en tres (I4,3\%). Se encontraron otros antecedentes quirúrgicos en diez (47,6\%) pacientes, los cuales incluían colecistectomías, cirugías estéticas, trasplantes renales, fistulotomías perianales, cuadrantectomías de mama y resecciones de quistes mamarios (tabla 2).

Tres (I4,3\%) pacientes no tenían ningún antecedente quirúrgico asociado con los implantes de endometriosis en la pared abdominal y $18(85,7 \%)$ presentaban lesión sobre una cicatriz quirúrgica previa. Las lesiones se localizaron predominantemente en la región inguinal (figura I).

La frecuencia de dolor fue de $95,2 \%$ y sus características se presentan en la figura $2 . \mathrm{La}$ mediana de la escala análoga del dolor fue 8,5 (RIQ=6-9). La sensación de masa se reportó en I9 (90,5\%) pacientes, de las cuales I6 $(84,2 \%)$ presentaron crecimiento de la lesión, $8(42, \mathrm{I} \%)$ reportaron el dolor como cíclico y otras ocho como constante. Dos $(9,5 \%)$ pacientes presentaron secreción en el sitio de la lesión, la cual fue de color rojo en una paciente y rojo-café en otra. En las dos pacientes, las lesiones eran umbilicales y la secreción de características cíclicas. La mediana del tiempo desde la cirugía hasta la aparición de la lesión fue de 36 meses (RIQ=I2-60) y la mediana del tiempo desde la aparición de la lesión hasta el diagnóstico fueron 24 meses (RIQ=6-60). En todas las pacientes, la enfermedad se presentó durante su vida fértil.
Tabla 2. Antecedentes quirúrgicos en 21 pacientes con endometriosis en la pared abdominal $(\mathrm{N}=21)$

\begin{tabular}{lc}
\hline Antecedentes quirúrgicos & $\mathbf{n ~ ( \% )}$ \\
\hline Cesárea & $15(71,4)$ \\
Laparoscopia ginecológica & $10(47,6)$ \\
Histerectomía & $7(33,3)$ \\
Episiotomía & $3(14,3)$ \\
Herniorrafia umbilical & $3(14,3)$ \\
Herniorrafia inguinal & $0(0,0)$ \\
Otros & $10(47,6)$ \\
\hline
\end{tabular}

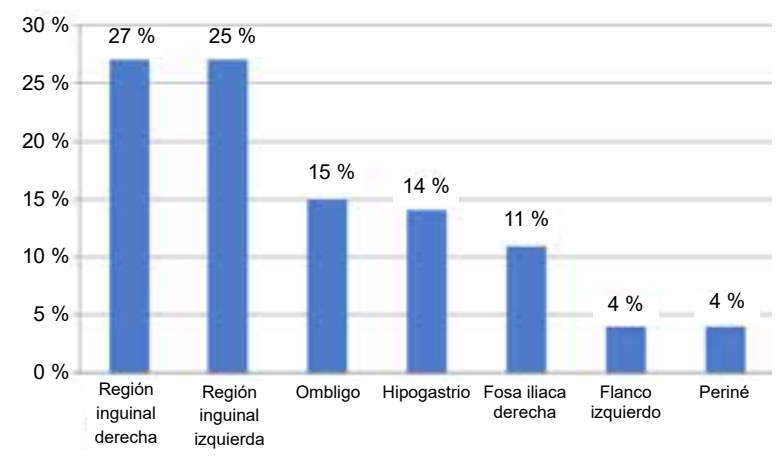

Figura 1. Localización de las lesiones endometriales en la pared abdominal

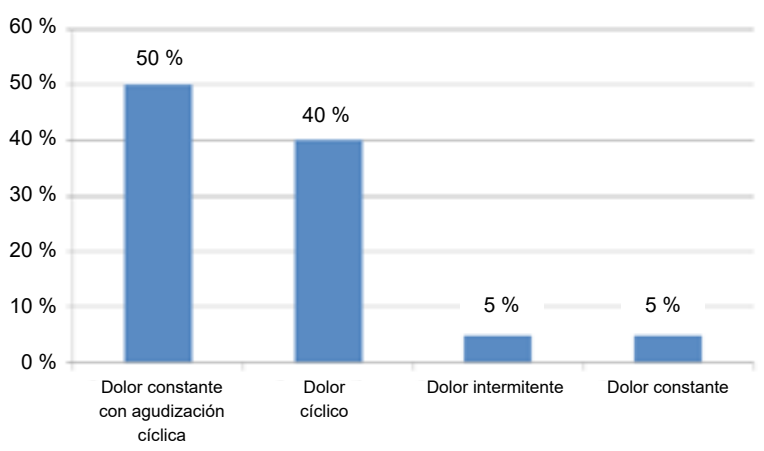

Figura 2. Característica del dolor en pacientes con endometriosis en la pared abdominal

Entre los diagnósticos diferenciales, se reportaron hernia de pared abdominal en tres pacientes (I4,3\%), tumor benigno en tres (I4,3\%), lipoma en dos (9,5\%), granuloma en dos (9,5\%), quiste subcutáneo en una paciente $(4,8 \%)$ y mioma en una paciente $(4,8 \%)$. Cinco $(23,8 \%)$ pacientes no tenían diagnóstico previo y cuatro (19,0 \%) tenían diagnóstico prequirúrgico de endometriosis de la pared abdominal. 
Entre los estudios diagnósticos utilizados, el más destacado fue la ecografía. Esta se practicó en I6 $(76,2 \%)$ pacientes y fue diagnóstica en una. A tres (I4,3\%) pacientes se les practicó BACAF, que permitió el diagnóstico en todas ellas. En esta serie no se hizo el diagnóstico mediante tomografía computarizada ni resonancia magnética.

El tratamiento fue sólo quirúrgico en I6 $(76,2 \%)$ pacientes, de las cuales diez mejoraron completamente sin presencia de recaídas. Cinco (23,8\%) pacientes recibieron tratamiento quirúrgico más tratamiento hormonal, de las cuales dos presentaron recaídas. A una paciente se le inició el tratamiento hormonal sin obtener mejoría, por lo que fue sometida a cirugía. El tiempo promedio del tratamiento hormonal fue de $\mathrm{I} 4$ meses ( $\mathrm{DE}=9,3)$. Inicialmente, 20 (95,2 \%) pacientes mejoraron con el tratamiento recibido; sin embargo, se documentó recidiva en el $38, \mathrm{I} \%$ de los casos.

\section{Discusión}

En la revisión más grande de pacientes con endometriosis de la pared abdominal, se incluyeron 445 pacientes de diferentes publicaciones y se encontró que la edad promedio había sido de de 3I años ', un poco menor que la del presente trabajo, que fue de 35,3 años. Los principales síntomas fueron sensación de masa (96\%), dolor (87\%) y cambios cíclicos (57\%) ․ El $20 \%$ fueron lesiones espontáneas que no se asociaron con cicatrices quirúrgicas previas, el $57 \%$ se asociaba con cicatrices de cesáreas, el II \%, con cicatrices de histerectomías y, el I3\%, con otras cicatrices de procedimientos quirúrgicos, como episiotomías y puertos de laparoscopia .

La mayoría $(87,9 \%)$ de las pacientes presentaron una masa dolorosa cerca o sobre una cicatriz quirúrgica previa ${ }^{6-8}$. En el presente estudio los resultados fueron similares, y se encontró dolor en el 95,2\% de las pacientes, con una mediana en la escala análoga de 8,5. Además, el 90,5\% de las pacientes reportó sensación de masa y, el $84.5 \%$, su crecimiento. En el 42,I \% de las pacientes, el dolor era de presentación cíclica, una tasa menor comparada a la de $89 \%$ reportada en el 2016 por Thornton, et al ${ }^{9}$. También, se encuentra descrita la secreción sanguinolenta por la lesión, la cual puede localizarse en la región umbilical ${ }^{2,6,10}$. En este estudio, se encontraron dos pacientes con secreción por la lesión, también de características cíclicas y de localización umbilical.

En el trabajo de Horton, et al., ${ }^{1}$ el $57 \%$ presentó lesiones sobre cicatrices de cesárea. Recientemente, Flórez, et al., " reportaron el 70 \% de las lesiones con igual ubicación.

Entre otros antecedentes quirúrgicos reportados en la literatura, se encuentran: laparoscopia diagnóstica, episiotomía, histerectomía, y herniorrafia umbilical e inguinal ${ }^{\mathrm{I}, \mathrm{II}}$.

Jamabo, et al., en su estudio publicado en el 2008, encontraron que el $36 \%$ de las pacientes tenían antecedentes de laparotomía exploratoria por útero perforado secundario a abortos ilegales 7; en otro trabajo publicado por Nominato, et al., encontraron que el $26,3 \%$ de las pacientes tenía lesiones en el periné posteriores a episiotomía ${ }^{12}$.

En el presente estudio, el $85,7 \%$ de las pacientes tenía la lesión en una cicatriz quirúrgica previa y, en el 7I,4\% por cesárea. Entre los otros antecedentes quirúrgicos, se destacan la laparoscopia diagnóstica y la histerectomía abdominal.

Se encuentra reportado que entre el $18,5 \mathrm{y}$ el $20 \%$ de las pacientes no presentaban antecedentes quirúrgicos, casos denominados espontáneos ${ }^{\mathrm{I}, \mathrm{II}, 13}$. En este estudio se encontró dicha presentación en el I4,3 \% de los casos. Recientemente, Touleimat, et al., ${ }^{14}$ en su trabajo de 23 pacientes, reportaron un tiempo promedio de 48 meses entre la cesárea y la aparición de endometriosis de la pared abdominal; sin embargo, Ecker, et al., ${ }^{\text {13 }}$ reportaron en 65 pacientes un tiempo promedio de 7 años entre la cirugía inicial y la aparición de la lesión. En el presente estudio, se encontró una mediana entre la cirugía índice y la aparición de la lesión de 36 meses, y la mediana entre la aparición de la lesión y su diagnóstico histopatológico fue de 24 meses.

Existen reportes de compromiso umbilical por endometriosis ${ }^{6,0,15,16}$. Generalmente este tipo de lesiones son espontáneas y se denominan nódulo 
de Villar. Victory, et al., en su revisión de la literatura de I22 pacientes con endometriosis umbilical, encontraron que solo el I4,7 \% tenía diagnóstico de endometriosis pélvica y, solo el I8 \%, antecedentes de cirugía abdominopélvica; de estas, el $40 \%$ tenía antecedentes de laparoscopia transumbilical. Además, alrededor del $50 \%$ de estas lesiones umbilicales presentaron sangrado cíclico ${ }^{17}$.

En este estudio, se encontraron resultados similares: cuatro pacientes con lesiones umbilicales, de las cuales dos tenían secreción de la lesión y eran de comportamiento cíclico. Una de las pacientes no tenía antecedentes quirúrgicos. Los tres casos asociados con cirugía tenían antecedentes de endometriosis pélvica.

En la revisión de la literatura de Victory, et al., el 67,6 \% fueron sometidas a resección quirúrgica, de las cuales una tuvo recaída ${ }^{17}$. En el presente trabajo, las cuatro pacientes fueron tratadas con resección quirúrgica y, de estas, tres presentaron recaída, probablemente explicado por inadecuados márgenes de resección.

La endometriosis del canal inguinal también se encuentra descrita por algunos autores ${ }^{\mathrm{IO}}$. En la presente serie, todas las pacientes que tenían implantes en la región inguinal presentaban antecedentes de incisión de Pfannenstiel y las lesiones se encontraron en la parte más lateral de la cicatriz.

En cuanto a la relación de la endometriosis de la pared abdominal con la endometriosis pélvica, esta última se encontró en el 38,I \% de las pacientes. Sin embargo, en otros trabajos, como el de Fernández, et al., de 15 pacientes, el $73 \%$ tenía compromiso ovárico concomitante ${ }^{18}$. En general, la incidencia de endometriosis pélvica en pacientes con endometriosis de la pared abdominal es similar entre ellas en mujeres en edad fértil, entre $8 \%$ y $15 \%$.

Los diagnósticos diferenciales descritos en la literatura incluyen hernias, hematomas, linfadenopatías, linfomas, lipomas, abscesos, quistes subcutáneos, granulomas, neuromas, sarcomas, tumores desmoides y lesiones metastásicas ${ }^{1,7,19}$. En este estudio, los diagnósticos diferenciales más comunes fueron hernias, tumores benignos, lipomas y granulomas. El diagnóstico prequirúrgico correcto de endometriosis de la pared abdominal, está descrito entre el 22 y el $47 \%$ en los diferentes reportes ${ }^{2,19}$. En este estudio, el diagnóstico antes del tratamiento fue correcto en el $19 \%$.

El tratamiento de elección es la escisión local amplia y con márgenes negativos ${ }^{19}$. Algunos autores recomiendan márgenes entre $5 \mathrm{y}$ Io $\mathrm{mm}^{20}$.

Todas las pacientes del presente estudio recibieron tratamiento con resección quirúrgica, ya fuera con tratamiento hormonal concomitante o sin él. El 95,2 \% mejoró con el tratamiento, pero hubo en total ocho recaídas que representan el $38, \mathrm{I} \%$ de la muestra, un número muy superior al de recurrencia de $4,3 \%$ reportada por Horton, et al., '; esto podría explicarse porque el 8I \% de las pacientes no presentaba diagnóstico prequirúrgico de endometriosis de la pared abdominal y, probablemente, se les practicó una resección con márgenes inadecuados.

Recientemente, Vitral, et al., ${ }^{21}$ publicaron un reporte de caso de endometriosis de la pared abdominal no palpable clínicamente, en el que se realizó marcación prequirúrgica de la lesión mediante la técnica de ROLL ${ }^{\mathrm{TM}}$ (Radioguided Occult Lesion Localization) con el uso de tecnecio-99m, lográndose una resección de la lesión con adecuados márgenes.

Como alternativas a la resección quirúrgica, además, se encuentran descritas técnicas menos invasivas, como la ablación con ultrasonido de alta intensidad, la radiofrecuencia y la crioablación ${ }^{20,22,23}$. Recientemente, Zhao, et al., ${ }^{23}$ reportaron un estudio comparativo entre la resección quirúrgica y la ablación por ultrasonografía de alta intensidad, y encontraron resultados similares en la remisión y la recurrencia del dolor después de un promedio de 32 meses de seguimiento. Por otro lado, Maillot, et al., ${ }^{24}$ llevaron a cabo un estudio comparativo entre el tratamiento con crioablación y la cirugía, y encontraron, a los 12 y 24 meses, tasas de ausencia de sintomatología del Ioo \% y el 66,7 \% ( IC $\left._{95 \%} 5,4-94,5\right)$, respectivamente, para el grupo de crioablación y $92 \%$ para el grupo cirugía en ambos puntos 
de tiempo $(\mathrm{p}=0,45)$; propusieron la crioablación como posible alternativa al tratamiento quirúrgico, sobre todo para lesiones múltiples y recidivas tras resecciones quirúrgicas previas.

Este estudio nos permite identificar las variables clínicas más comunes en las pacientes con endometriosis de la pared abdominal y, así, lograr una sospecha clínica temprana.

Los datos de este trabajo se recolectaron por medio de entrevistas telefónicas. De esta forma, se logró recolectar información precisa sobre la sintomatología de la enfermedad y su cronología, información que podría haber estado excluida de las historias clínicas, teniendo en cuenta el difícil diagnóstico prequirúrgico, según lo reportado en la literatura. Además, la muestra de pacientes fue tomada de un laboratorio a donde llegan muestras de patología de múltiples instituciones, lo que hacía muy difícil la recolección de las historias clínicas. Este diseño tiene la ventaja de permitir analizar muy bien la enfermedad actual de cada paciente, pero hace que no se puedan obtener los datos de laboratorio, los reportes de imágenes o las descripciones quirúrgicas. Todos los datos analizados provienen de las respuestas proporcionadas por las pacientes, las cuales pueden tener exactitud variable, sobre todo en los datos de tiempo. Además, tiene todas las limitaciones de los estudios retrospectivos y descriptivos que no permiten establecer asociaciones entre variables, pero la baja incidencia de la enfermedad hace difícil adelantar estudios controlados.

\section{Conclusión}

La endometriosis de la pared abdominal es uno de los diagnósticos que se deben considerar en aquellas mujeres con masas de la pared abdominal y que el cirujano debe manejar dentro su marco teórico. Es una enfermedad que rara vez se diagnostica antes de los resultados de histopatología, por lo cual presenta retrasos en su diagnóstico y alteración de la calidad de vida de estas pacientes. La presencia de una masa dolorosa en una cicatriz de origen ginecobstétrico es una de las principales características de esta entidad.
Una vez establecido el diagnóstico, se debe garantizar una adecuada resección quirúrgica, considerada hoy por hoy como la mejor alternativa de tratamiento.

\section{Cumplimiento de normas éticas}

Consentimiento informado: Este estudio es una revisión retrospectiva de informes de patología y entrevista telefónica, y como tal, no hay necesidad de un consentimiento informado por escrito. El Comité de Ética Institucional aprobó el diseño y la metodología del estudio.

Conflictos de interés: ninguno reportado por los autores.

Fuente de financiación: autofinanciado por los autores.

Agradecimientos: los autores agradecen a Laboratorio y Ayudas Diagnósticas Dinámica, por aportar la base de datos de las pacientes del estudio.

\section{Referencias}

I. Horton JD, DeZee KJ, Ahnfeldt EP, Wagner M. Abdominal wall endometriosis: A surgeon's perspective and review of 445 cases. Am J Surg. 2008;196:207-I2. doi: I0.IOI6/j.amjsurg.2007.07.035

2. Bektas H, Bilsel Y, Sari YS, Ersöz F, Koç O, Deniz M, et al. Abdominal wall endometrioma; A ro-year experience and brief review of the literature. J Surg Res. 20I0;I64:77-8I. doi: I0.IOI6/j.jss.20I0.07.043

3. Rindos NB, Mansuria S. Diagnosis and management of abdominal wall endometriosis: A systematic review and clinical recommendations. Obstet Gynecol Surv. 2017;72:II6-22. doi: I0.I097/OGX.oooooooooooo0399

4. Farooq U, Laureano AC, Miteva M, Elgart GW. Cutaneous endometriosis: Diagnostic immunohistochemistry and clinicopathologic correlation. J Cutan Pathol. 20II;38:525-8. doi: I0.IIII/j.I600-0560.20II.0I68I.x

5. Khan Z, Zanfagnin V, El-Nashar SA, Famuyide AO, Daftary GS, Hopkins MR. Risk factors, clinical presentation, and outcomes for abdominal wall endometriosis. J Minim Invasive Gynecol. 2017;24:478-84. doi: I0.I0I6/j.jmig.20I7.0I.005

6. Leite GK, Carvalho LF, Korkes H, Guazzelli TF, Kenj G, Viana Ade T. Scar endometrioma following obstetric surgical incisions: Retrospective study on 33 cases and 
review of the literature. Sao Paulo Med J. 2009;127:270-7.

7. Jamabo RS, Ogu RN. Abdominal scar endometriosis. Indian J Surg. 2008;70:I84-7. doi: I0.1007/sI2262-008o050-8

8. Cöl C, Yilmaz EE. Cesarean scar endometrioma: Case series. World J Clin Cases. 20I4;2:133-6. doi: I0.12998/ wjcc.v2.i5.133

9. Thornton S, Woll J, Markfeld-Erol F, Hasenburg A, Proempeler H, Bossart M. Abdominal wall endometriosis after gynaecological interventions - A cohort study on diagnostic and treatment of abdominal wall endometriosis. Int J Surg Res Pract. 2016;3:044. doi:I0.23937/2378-3397/I4IOO44

Io. Agarwal A, Fong YF. Cutaneous endometriosis. Singapore Med J. 2008;49:704-9.

II. Florez ML, Jiménez D, Rojo M, Camarero E, Saez P, Cabeza J, et al. Endometriosis de la pared abdominal: experiencia en nuestro centro. Cir Esp. 2018;96:7.

I2. Nominato NS, Prates LF, Lauar I, Morais J, Maia L, Geber S. Caesarean section greatly increases risk of scar endometriosis. Eur J Obstet Gynecol Reprod Biol. 20I0;I52:83-5. doi: I0.IoI6/j.ejogrb.20I0.05.00I

I3. Ecker AM, Donnellan NM, Shepherd JP, Lee TT. Abdominal wall endometriosis: I2 years of experience at a large academic institution. Am J Obstet Gynecol. 20I4;2II:363.eI-5. doi: IO.IOI6/j.ajog.20I4.04.0II

I4. Touleimat S, Darwish B, Vassilieff M, Stochino Loi E, Hennetier C, Roman H. Abdominal wall endometriosis following cesarean section: A study of the growth rate of parietal endometriosis implants. Minerva Ginecol. 2017;69:440-6. doi: 10.23736/Soo26-4784.17.0403I-X

I5. Seidel A, Ruiz OA. Nódulo de Villar. Presentación de un caso. Rev Asoc Col Dermatol. 2009;17:48-50.

I6. Jaime TJ, Jaime TJ, Ormiga P, Leal F, Nogueira OM, Rodríguez N. Umbilical endometriosis: Report of a case and its dermoscopic features. An Bras Dermatol.
20I3;88:I2I-4.

I7. Victory R, Diamond MP, Johns DA. Villar's nodule: A case report and systematic literature review of endometriosis externa of the umbilicus. J Minim Invasive Gynecol. 2007;I4:23-32.

I8. Fernández-Aceñero MJ, Córdova S. Cutaneous endometriosis: Review of 15 cases diagnosed at a single institution. Arch Gynecol Obstet. 20II;283:IO4I-4. doi: I0.IOO7/s00404-OIO-I484-3

19. Mejía FJ, Abadía HA, Vélez A, Restrepo HD. Endometriomas extrapélvicos. Med UPB. I997;16:I49-56.

20. Cornelis F, Petitpierre F, Lasserre AS, Tricaud E, Dallaudière B, Stoeckle E, et al. Percutaneous cryoablation of symptomatic abdominal scar endometrioma: Initial reports. Cardiovasc Intervent Radiol. 20I4;37:1575-9. doi: I0.1007/s00270-0I4-0843-z

2I. Vitral GSF, Salgado HC, Rangel JMC. Use of radioguided surgery in abdominal wall endometriosis: An innovative approach. World J Nucl Med. 2018;17:204-6. doi: I0.4I03/wjnm.WJNM_47_I7

22. Carrafiello G, Fontana F, Pellegrino C, Mangini M, Cabrini L, Mariani D, et al. Radiofrequency ablation of abdominal wall endometrioma. Cardiovasc Intervent Radiol. 2009;32:1300-3. doi: I0.I007/s00270-008-9500-8

23. Zhao L, Deng Y, Wei Q, Chen J, Zhao C. Comparison of ultrasound-guided high-intensity focused ultrasound ablation and surgery for abdominal wall endometriosis. Int J Hyperthermia. 2018;35:528-533. doi: I0.1080/02656736.2018.1511836

24. Maillot J, Brun JL, Dubuisson V, Bazot M, Grenier N, Cornelis FH. Mid-term outcomes after percutaneous cryoablation of symptomatic abdominal wall endometriosis: Comparison with surgery alone in a single institution. Eur Radiol. 2017;27:4298-306. doi: I0.IO07/ soo330-0I7-4827-7 